hep-th/9707247

SNUTP/97-106

July 1997

\title{
Superpotentials of $N=1$ Supersymmetric Gauge Theories from M-theory
}

\author{
Soonkeon $\mathrm{Nam}^{\S}$, Kyungho $\mathrm{Oh}^{*}$, Sang-Jin $\mathrm{Sin}^{\dagger}$ \\ $\S$ Dept. of Physics, Kyung Hee University, Seoul 130-701, Korea \\ nam@nms . kyunghee. ac. $\mathrm{kr}$ \\ * Dept. of Mathematics, University of Missouri-St. Louis, \\ St. Louis, Missouri 63121, USA \\ oh@arch.umsl.edu \\ $\dagger$ Dept. of Physics, Hanyang University, Seoul 133-791, Korea \\ sjs@dirac.hanyang.ac. $\mathrm{kr}$
}

\begin{abstract}
We consider brane configurations in M-theory describing $N=1$ supersymmetric gauge theories and using the parametric representation of the brane configurations, we calculate the superpotentials for various cases including multiple gauge groups or fermions. For $S U(n) N=1 \mathrm{SQCD}$ with $N_{f}$ fermion case $\left(N_{f}<N_{c}\right)$, we find that the superpotential from M-theory and the gauge theory agree precisely. This gives a direct evidence of the validity of Witten's M-theory method for calculating the superpotential.
\end{abstract}




\section{Introduction}

The idea of D-brane [1] opened up a new and surprisingly simple way to communicate between super symmetric (SUSY) gauge theories and the superstring theories. Many intriguing results about the field theories have been achieved by investigating gauge theories formulated on the world-volume of branes in string theories[2-11] and Mtheory[12-16]. In a recent paper [12], Witten provided solutions of $N=2$ SUSY gauge theories in four dimension[17, 18] by reinterpreting configurations of fourbranes and fivebranes in type II superstring as the branes in M theory. Moreover, in a subsequent paper 15], he showed how some of the outstanding problems in particle physics such as quark confinement and chiral symmetry breaking, can be approached from the Mtheory point of view. There, he also suggested a way to calculate the superpotential directly from a brane configuration, thereby he gave a direct evaluation of the tension of the domain wall 19. Though very insightful, no direct comparison with the gauge theory result was given.

One of the main goal of this paper is to give a direct evidence for the validity of Witten's method by extending his result to the cases where gauge theory results are available. We will show that the minimum of the superpotential of the $N=1$ $S U(n)$ gauge theory superpotential agrees with the value evaluated with the M-theory method. We will also generalize and obtain the superpotentials for more general situations with product gauge groups, which corresponds to the brane configurations where there are $n+1$ fivebranes. To motivate the study of multiple branes we recall the works of Elitzur et al. who have considered $k$ 'coincident' NS fivebranes linked to an 'coincident' NS fivebrane linked by $N_{c}$ D fourbranes [5].

\section{The Brane Configuration}

To fix the notation, we begin with brane configurations in type IIA superstring theory. Let the spacetime coordinates be $\left(x^{0}, x^{1}, \ldots, x^{9}\right)$ on ${ }^{10}$. Our basic brane configuration contains three kinds of branes: NS fivebranes whose world volume occupies $\left(x^{0}, x^{1}, x^{2}, x^{3}, x^{4}, x^{5}\right)$ spacetime coordinates, D fourbranes occupying $\left(x^{0}, \cdots, x^{3}, x^{6}\right)$, 
and D sixbranes occupying $\left(x^{0}, \cdots, x^{3}, x^{7}, x^{8}, x^{9}\right)$.

The basic brane configuration consists of $N_{c}$ fourbranes suspended between two parallel fivebranes in Type IIA superstring theory which gives a representation of $N=2$ SUSY QCD (SQCD) in four dimensions $\left(x^{0}, \cdots, x^{3}\right)$ with gauge group $S U\left(N_{c}\right)$. Also $N_{f}$ D sixbranes could be added and this gives $N_{f}$ hypermultiplets in the fundamental representation of the gauge group $S U\left(N_{c}\right)$.

One of the limitation of type IIA superstring theory is that a fourbrane ending on a fivebrane defies any explicit conformal field theory description. However, by going to $\mathrm{M}$ theory, the picture becomes more unified. A type IIA fourbrane becomes an $\mathrm{M}$ theory fivebrane that is wrapped over the $11^{\text {th }}$ dimension ${ }^{1}$. Thus $\mathrm{D}$ fourbranes and NS fivebranes come from the same basic object in M theory. This $N=2$ SUSY description can be broken to $N=1$ by turning on a mass for the adjoint chiral superfield in the $N=2$ vector multiplet.

Let us now review some of the Witten's construction 12, 15 of the parametric representation of the brane configuration in $\mathrm{M}$ theory for $N=1 \mathrm{SQCD}$. To set up the brane configurations in complex geometry, we introduce complex coordinates $v=x^{4}+i x^{5}, w=x^{7}+i x^{8}, t=\exp \left(-\left(x^{6}+i x^{10}\right) / R\right)$. The brane configuration for $S U(n)$ gauge theory is given by the Seiberg-Witten curve 17]

$$
t^{2}+P_{n}(v) t+1=0
$$

in $v-t$ space when $w=0$. Here $P_{n}(v)$ is a polynomial of the form

$$
P_{n}(v)=v^{n}+u_{2} v^{n-2}+\ldots+u_{n}
$$

where the $u_{i}$ 's are the "order parameters" of the theory.

The brane configuration associated to $N=1$ SUSY can be obtained by "rotating" the brane configuration that describes $S U(n)$ gauge theory with $N=2$ SUSY, in the sense of Ref. 21]. The rotation is possible only when all the 1-cycles on the corresponding curve are degenerate. This is why the $N=1$ curve is rational for the two NS fivebrane case 15. However for more than two NS fivebranes, we can ask whether the curve is generically rational. By reversing the argument we can say 
that $N=2$ SUSY forbids the rotation of the NS fivebranes. Once $N=2$ SUSY is broken, however, there is nothing that freezes the rotating degrees of freedom. This means that in generic configuration the NS fivebranes are rotated to one another by certain angles. Therefore the curves with positive genus correspond very special non-generic cases. Hence the generic M-brane configuration corresponds to a curve $\Sigma$ of genus zero, which has parametric representations. Namely, $\Sigma$ can be identified by a holomorphic mapping with a Riemann sphere with some points deleted. If $\lambda$ is the complex coordinate of $\Sigma$, the holomorphic functions $v(\lambda), w(\lambda)$, and $t(\lambda)$ on $\Sigma$ are given by rational functions of $\lambda$.

Since $v$ has only poles at ends of fivebranes, we can put $v=\lambda+c \lambda^{-1}$, for some constant $c$. Also, $t$ can go to zero or infinity only at poles of $v$, so $t$ is a constant multiple of a power of $\lambda$, i.e. $t \sim \lambda^{n}$. We also get $c^{n}=1$ from eq.(1), and for each choice of $c$ the polynomial $P_{n}$ is uniquely determined. After the rotation, $w$, instead of being zero, should be a non-zero holomorphic function on $\Sigma$. Rotating only one fivebrane, $w$ should get a pole only at one end of $\Sigma$ and should vanish at the other end. So we can set $w=\zeta \lambda^{-1}$ for some complex constant $\zeta$. As $\lambda$ goes to zero $w / v \rightarrow \zeta / c$, therefore it can be regarded as a tangent of the rotation angle, namely

$$
\tan \theta=|\zeta / c|
$$

For the rotation of $\theta=\pi / 2$, one should set $c=0$, because $v$ and $w$ do not have a common pole. Therefore the parametric equation for the curve becomes; $v=\lambda$, $w=\zeta \lambda^{-1}, t=\lambda^{n}$.

Now we give a parametric representation of the brane configuration with more than two NS fivebranes. Suppose NS fivebranes are located at $\lambda=\lambda_{\alpha}, \alpha=0,1, \cdots, n$ at arbitrary angles in $v-w$ space, with $k_{\alpha}$ D fourbranes suspended between $\alpha-1^{\text {th }}$ and $\alpha^{\text {th }}$ fivebranes. Thus the functions $v$ and $w$ on $\Sigma$ have poles at $\lambda_{\alpha}, \alpha=0,1, \cdots, n$. The function $t$ on $\Sigma$ will have a zero or pole according to the direction the NS fivebrane is bent. If there are more $\mathrm{D}$ fourbranes in the left than right it will bend to the right, therefore it will have a zero and vice versa. In fact $t \sim v^{a_{\alpha}}$ as $v \rightarrow \infty$ where 
$a_{\alpha}=k_{\alpha+1}-k_{\alpha}$. Putting all these together, we have

$$
\begin{aligned}
& v=\sum_{\alpha=0}^{n} \frac{c_{\alpha}}{\lambda-\lambda_{\alpha}} \\
& w=\sum_{\alpha=0}^{n} \frac{s_{\alpha}}{\lambda-\lambda_{\alpha}} \\
& t=\prod_{\alpha=0}^{n}\left(\lambda-\lambda_{\alpha}\right)^{-a_{\alpha}}
\end{aligned}
$$

for some complex numbers $c_{\alpha}, s_{\alpha}$. We normalize the system by setting $c_{0}=1, s_{0}=0$, so that $\lambda_{0}$ is the location of the unrotated NS fivebrane. Then the rotation angles $\theta_{\alpha}$ 's are again given by

$$
\tan \theta_{\alpha}=\left|\frac{s_{\alpha}}{c_{\alpha}}\right|
$$

Notice that Witten's choice corresponds to the special case where two poles are chosen as $\lambda=0$ and $\infty$ and with rotation angle $\pi / 2$. Since it can be related to the $n=1$ case of above with $\left(\lambda_{0}, \lambda_{1}\right)=(0,1)$ by the Möbius transformation $\lambda \rightarrow \tilde{\lambda}=\frac{\lambda-1}{\lambda}$ followed by constant shifts in $v$ and $w$, they are physically equivalent.

When one approaches the ends of the fivebranes, $v$ and $w$ go to infinity. Since the effect of the rotation can be ignored, the equation for the $N=2$ Seiberg-Witten curve

$$
t^{n+1}+P_{k_{1}}(v) t^{n}+\cdots+1=0
$$

must be satisfied near the ends. This determines some of the moduli of the $N=1$ curve in terms of the moduli of the polynomial equation for $N=2$ case. As $\lambda$ approaches $\lambda_{\alpha}$ the parametrization of the curve is asymptotically given by,

$$
\begin{aligned}
& v \rightarrow \frac{c_{\alpha}}{\left(\lambda-\lambda_{\alpha}\right)}, \\
& w \rightarrow \frac{s_{\alpha}}{\left(\lambda-\lambda_{\alpha}\right)}, \\
& t \rightarrow\left(\lambda-\lambda_{\alpha}\right)^{-a_{\alpha}} \prod_{\beta \neq \alpha}\left(\lambda_{\alpha}-\lambda_{\beta}\right)^{-a_{\beta}} .
\end{aligned}
$$

Inserting these to eq.(6), we get the following:

$$
c_{\alpha}^{a_{\alpha}}=-\frac{p_{\alpha+1}}{p_{\alpha}} \prod_{\beta \neq \alpha}\left(\lambda_{\alpha}-\lambda_{\beta}\right)^{-a_{\beta}}, \quad(\alpha=0, \cdots, n)
$$


where $p_{\alpha}$ denotes the coefficient of the leading power in $P_{k_{\alpha}}(v)=p_{\alpha} v^{k_{\alpha}}+\cdots$. This determines the coefficients of the poles of $v$ as function of the location of the fivebranes and the information of $N=2$ theory up to the phase $e^{2 \pi i /\left(k_{\alpha+1}-k_{\alpha}\right)}$. As Witten argued, $p_{\alpha}$ 's are parameters of the Lagrangian rather than a modulus, so we conclude that only the locations of the NS fivebranes $\lambda_{\alpha}$ 's and the parameter for angles of the $\alpha+1^{\text {th }}$ NS brane relative to the first one, $s_{\alpha}$ 's, are the moduli of the $N=1$ theory.

Let us now discuss the symmetry of the curve. Although there is no remaining symmetry for interacting branes, asymptotically, when $\lambda$ is close to $\lambda_{\alpha}$, we have following symmetry:

$$
\prod_{\alpha=1}^{n} \mathbf{Z}_{\left|a_{\alpha}\right|}=\mathbf{Z}_{k_{2}-k_{1}} \times \cdots \times \mathbf{Z}_{k_{n-1}-k_{n}} \times \mathbf{Z}_{k_{n}} .
$$

This corresponds to the rotational symmetries of the branes when $v$ goes to infinity broken to the discrete symmetries due to the quantum effects. We remark that this is the analogue of chiral symmetry for $S U(n)$ case in which the chiral symmetry is $\mathbf{Z}_{n}$. The structure of the symmetry manifestly reveal the consequence of the interactions between the branes. The field theory analogues of this effect for the product gauge groups are not fully studied. One technical remark is that in considering the symmetry properties, it is convenient to locate the zeroth brane at the infinity so that it looks special relative to the others.

\section{The Superpotential}

Given the brane configurations in M-theory, we calculate the superpotential of the corresponding gauge theory following Witten's idea 15]. Consider in general M-theory compactification on $\mathbf{R}^{4} \times X \times \mathbf{R}$ where $X$ is a Calabi-Yau threefold. Suppose in spacetime there are fivebranes of the form $\mathbf{R}^{4} \times \Sigma, \Sigma$ being a two-dimensional real surface in $X$. Choose $\Sigma_{0}$ in the homology class of $\Sigma$ in $H^{2}(X, \mathbf{R})$. Then there exists a three manifold $B$ and a map $\Phi_{B}: B \rightarrow X$ such that the boundary of $B$ maps $\Sigma-\Sigma_{0}$ in $X$. Let $\Omega$ be the holomorphic three form on $X$. Then as Witten suggested, the 
superpotential is given by

$$
W(\Sigma)-W\left(\Sigma_{0}\right)=\int_{B} \Phi_{B}^{*}(\Omega) .
$$

This defines $W(\Sigma)$ up to an additive constant. The ambiguity in $W(\Sigma)$ comes from the choices of $B$ and $\Sigma_{0}$. In general, the periods of $\Omega$ also contribute to the ambiguity, but this indeterminancy disappears since $H_{3}(X)=$,0 in our applications. The condition $H_{3}(X)=$,0 also forces that the space of all possible $\Sigma$ is simply-connected. Thus a different choice of $B$ does not create any additional constant.

To discuss the issue of the $\Sigma_{0}$ dependence, we consider two NS fivebranes connected by $n \mathrm{D}$ fourbranes and assume that two are at a relative angle $\theta$. We take $X$ to be a flat Calabi-Yau manifold $Y$ with coordinates $v, w$, and $t$. The holomorphic three form $\Omega$ on $Y$ is given by

$$
\Omega=R \frac{d t}{t} \wedge d v \wedge d w
$$

It is chosen such that $\Omega \wedge \bar{\Omega}$ is the Riemannian volume form for $Y$. Since we can choose the position of a fivebrane at $\lambda=0$ without loss of generality, the configuration is parametrized by

$$
\begin{aligned}
& v(\lambda)=\frac{1}{\lambda-\lambda_{1}}+\frac{c}{\lambda}, \\
& w(\lambda)=\frac{\zeta}{\lambda} \\
& t(\lambda)=\left(\frac{\lambda}{\lambda-\lambda_{1}}\right)^{n} .
\end{aligned}
$$

In fact the dependence of the superpotential on the choice of the $\Sigma_{0}$ can be a subtle issue. In principle, fixing a $\Sigma_{0}$ just amounts to fixing the zero point of the superpotential. In practice, however, for a given $\Sigma$ one has to choose an appropriate $\Sigma_{0}$ to expedite calculation. Therefore we want to put $W\left(\Sigma_{0}\right)=0$ for a certain class of surfaces. In the next paragraph, for the fixed $\Sigma$ given by eq.(12), we choose $\tilde{\Sigma}_{0}$ and $\Sigma_{0}$ which look completely different, and show by explicit calculation that they in fact give the same superpotential.

To construct $\tilde{\Sigma}_{0}$ we first introduce a new variable $\tilde{\lambda}=\frac{\lambda}{\lambda-\lambda_{1}}$ and take $\Sigma_{\lambda}$ to be the complex $\lambda$-plane with 0 and $\lambda_{1}$ deleted. We write $\tilde{\lambda}=\exp (\tilde{\rho}+i \tilde{\theta})$, with $\tilde{\rho}$ and 
$\tilde{\theta}$ real, and pick an arbitrary smooth function $\tilde{f}$ of a real variable such that $\tilde{f}(\tilde{\rho})=1$ for $\tilde{\rho}>2$ and $\tilde{f}(\tilde{\rho})=0$ for $\tilde{\rho}<1$. Then we define the map $\Phi_{0}: \Sigma_{\lambda} \rightarrow Y$ by

$$
\begin{aligned}
v & =\frac{1}{\lambda-\lambda_{1}} \tilde{f}(\tilde{\rho})+\frac{c}{\lambda} \tilde{f}(-\tilde{\rho}), \\
w & =\tilde{f}(-\tilde{\rho}) \frac{\zeta}{\lambda} \\
t & =\left(\frac{\lambda}{\lambda-\lambda_{1}}\right)^{n} .
\end{aligned}
$$

By construction $\tilde{\Sigma}_{0}$ is asymptotic at infinity to $\Sigma$. We now introduce smooth bounded functions $g_{ \pm}=g_{ \pm}(\tilde{\rho}, \sigma)$ given by $g_{+}(\tilde{\rho}, 1)=1, g_{+}(\tilde{\rho}, 0)=f(\tilde{\rho})$, and $g_{+}(\tilde{\rho}, \sigma)=1$, and $g_{-}(\tilde{\rho}, \sigma)=g_{+}(-\tilde{\rho}, \sigma)$. The map $\Phi_{B}: B \rightarrow Y$ can then be defined by

$$
\begin{aligned}
v & =\frac{1}{\lambda-\lambda_{1}} g_{+}(\tilde{\rho}, \sigma)+\frac{c}{\lambda} g_{-}(\tilde{\rho}, \sigma), \\
w & =\frac{\zeta}{\lambda} g_{-}(\tilde{\rho}, \sigma) \\
t & =\tilde{\lambda}^{n}
\end{aligned}
$$

The superpotential now becomes

$$
W(\Sigma)-W\left(\tilde{\Sigma}_{0}\right)=R n \int_{B} \frac{d \tilde{\lambda}}{\tilde{\lambda}} \wedge d v \wedge d w
$$

and thus

$$
W(\Sigma)-W\left(\tilde{\Sigma}_{0}\right)=i R n \int_{0}^{1} d \sigma \int_{0}^{2 \pi} d \tilde{\theta} \int_{-\infty}^{\infty} d \tilde{\rho} \frac{\zeta}{\lambda_{1}^{2}} \frac{(\tilde{\lambda}-1)^{2}}{\tilde{\lambda}}\left(\frac{\partial g_{+}}{\partial \sigma} \frac{\partial g_{-}}{\partial \rho}-\frac{\partial g_{+}}{\partial \rho} \frac{\partial g_{-}}{\partial \sigma}\right)
$$

Notice that the $g_{ \pm}$is independent of $\tilde{\theta}$. Therefore the integrand is sum of the terms whose integrals split into $\int d \tilde{\theta}$ and the rests. The final result is

$$
W(\Sigma)=-\frac{4 \pi i R n \zeta}{\lambda_{1}^{2}} .
$$

Now we choose a different $\Sigma_{0}$ given by the $\Phi_{0}$ which is determined by continuous and smooth functions $f_{0}, f_{1}$. Let $\lambda / \lambda_{1}=\exp (\rho+i \theta)$ and construct $f_{i}$ 's such that $f_{0}(\rho)=1$ if $\rho<-3, f_{0}=0$ if $\rho>-2$ and $f_{1}(\rho)=1$ if $|\rho-1|<1, f_{1}=0$ if $|\rho-1|>2$. Construct the map $\Phi_{0}: \Sigma_{\lambda} \rightarrow Y$ just as above by replacing $\tilde{f}(\rho)$ and $\tilde{f}(-\rho)$ by $f_{i}(\rho)$ 's. Then construct $g_{i}, i=0,1$ such that they interpolate $f_{i}$ and 
constant function 1 continuously and smoothly. Then we choose the map $\Phi_{B}: B \rightarrow Y$ as above by replacing $g_{ \pm}$by $g_{i}$ 's. Then the superpotential now becomes

$$
W(\Sigma)-W\left(\Sigma_{0}\right)=i R n \int\left[\frac{1}{\lambda^{2}\left(\lambda-\lambda_{1}\right)}-\frac{1}{\lambda\left(\lambda-\lambda_{1}\right)^{2}}\right] d \lambda \wedge d g_{1} \wedge d g_{0}
$$

This integral also can be evaluated easily by noticing that $g_{i}$ 's do not depend on the angular variable $\theta$, and is exactly the same as the previous case. Although the bases of $f$ 's and $\tilde{f}$ 's, defined as the regions in $\Sigma_{\lambda}$ where $\Sigma_{0}$ and $\tilde{\Sigma}_{0}$ respectively is equal to $\Sigma$, look very different, $\Sigma_{0}$ and $\tilde{\Sigma}_{0}$ has a common property. They both asymptotically approach to $\Sigma$ and have necks that have zero thickness which makes the ${ }_{n}$ invariance more manifest. The general construction described below will respect this feature.

Notice that the superpotential is independent of the rotation angle for the two fivebrane cases. There are overall factor 2 difference between the previous result with that for Witten's configuration, which gives $2 \pi$ rather than $4 \pi$. This can be understood by noticing that in Witten's choice, the pole at the infinity is truncated from the expression of $t$ so that the contribution from that pole is not included.

Now let us calculate the superpotential for the general multi-brane cases, given by eq.(4). We assume that $\left|\lambda_{0}\right|>\left|\lambda_{1}\right|>\cdots>\left|\lambda_{n}\right|$. Let $\rho=\log |\lambda|, \rho_{\alpha}=\log \left|\lambda_{\alpha}\right|$ for $\alpha=0, \cdots, n$, and $\epsilon=\min _{\alpha=0, \ldots, n}\left(\rho_{\alpha}-\rho_{\alpha+1}\right)$. We construct $\Sigma_{0}$ in terms of the functions $f_{\alpha}(\rho)$ which are defined by

$$
\begin{aligned}
f_{\alpha}(\rho)=1 \quad \text { if } \quad\left|\rho-\rho_{\alpha}\right|<\frac{1}{3} \epsilon \\
=0 \quad \text { if } \quad\left|\rho-\rho_{\alpha}\right|>\frac{2}{3} \epsilon
\end{aligned}
$$

and $f_{\alpha}$ interpolate 0 and 1 in the region $\frac{1}{3} \epsilon<\left|\rho-\rho_{\alpha}\right|<\frac{2}{3} \epsilon$. We define the homotopy functions $g_{\alpha}(\rho, \sigma)$ 's which interpolate $f_{\alpha}(\rho)$ and 1 such that $g_{\alpha}(\rho, 0)=f_{\alpha}(\rho)$, $g_{\alpha}(\rho, 1)=1$. In other words, each $f_{\alpha}$ has a circular strip containing the circle passing the $\lambda_{\alpha}$ as its territory where it is 1 and rapidly dies outside and no territories are overlapping so that $f_{\alpha} f_{\beta}=0$ for any pair. Furthermore for later purpose we construct the $g_{\alpha}$ 's such that

$$
g_{\alpha}\left(\rho_{\gamma}, \sigma\right)=g_{\beta}\left(\rho_{\gamma}, \sigma\right):=h_{\gamma}(\sigma)
$$


for any triple $\alpha, \beta, \gamma$ which are different to one another. Now take a three manifold $B$ as the product of the $\lambda$ plane with all the $\alpha$ 's deleted and the $\sigma$ interval $0 \leq \sigma \leq 1$. Then define a map $\Phi: B \rightarrow Y$ by

$$
\begin{aligned}
& v=\sum_{\alpha=0}^{n} \frac{c_{\alpha}}{\lambda-\lambda_{\alpha}} g_{\alpha}(\rho, \sigma), \\
& w=\sum_{\alpha=0}^{n} \frac{s_{\alpha}}{\lambda-\lambda_{\alpha}} g_{\alpha}(\rho, \sigma), \\
& t=\prod_{\alpha=0}^{n}\left(\lambda-\lambda_{\alpha}\right)^{-a_{\alpha}} .
\end{aligned}
$$

Now the superpotential can be written down as

$$
\begin{aligned}
W & =W(\Sigma)-W\left(\Sigma_{0}\right)=R \int \frac{d t}{t} \wedge d v \wedge d w \\
& =R \sum_{\alpha, \beta, \gamma} c_{\alpha} s_{\beta} a_{\gamma} \int d g_{\alpha} \wedge d g_{\beta} \wedge d \lambda I_{\alpha, \beta, \gamma}(\lambda),
\end{aligned}
$$

where

$$
I_{\alpha, \beta, \gamma}(\lambda)=\frac{1}{\left(\lambda-\lambda_{\alpha}\right)\left(\lambda-\lambda_{\beta}\right)\left(\lambda-\lambda_{\gamma}\right)} .
$$

Let $\lambda=|\lambda| e^{i \theta}$ and $z=e^{i \theta}$. Then we can first evaluate the $d \lambda$ integral as $d \theta$ integral, which in turn can be evaluated as contour integral on $z$ plane along the unit circle. Therefore

$$
\int d \lambda I_{\alpha, \beta, \gamma}(\lambda)=\int|\lambda| d z \frac{1}{\left(|\lambda| z-\lambda_{\alpha}\right)\left(|\lambda| z-\lambda_{\beta}\right)\left(|\lambda| z-\lambda_{\gamma}\right)} .
$$

Due to the presence of the homotopy factors, one can avoid $\lambda_{\alpha}=\lambda_{\beta}$ However, we can have $\lambda_{\gamma}=\lambda_{\alpha}$ or $\lambda_{\gamma}=\lambda_{\beta}$.

- The case with $\alpha \neq \gamma \neq \beta$

$$
\int d \lambda I_{\alpha, \beta, \gamma}(\lambda)=2 \pi i\left[\Lambda_{\alpha} \Theta\left(\rho-\rho_{\alpha}\right)+\Lambda_{\beta} \Theta\left(\rho-\rho_{\beta}\right)+\Lambda_{\gamma} \Theta\left(\rho-\rho_{\gamma}\right)\right],
$$

where $\Lambda_{i}$ is the residue of $I_{\alpha, \beta, \gamma}(\lambda)$ at $\lambda_{i}$ with $i=\alpha, \beta, \gamma$, and $\Theta$ denotes the usual step function. 
- The cases $\gamma=\alpha$ or $\gamma=\beta$

$$
\begin{aligned}
\int d \lambda I_{\alpha, \beta, \alpha}(\lambda) & =\int|\lambda| d z \frac{1}{\left(|\lambda| z-\lambda_{\alpha}\right)^{2}\left(|\lambda| z-\lambda_{\beta}\right)} \\
& =\frac{1}{\lambda_{\alpha \beta}^{2}}\left[-\Theta\left(\rho-\rho_{\alpha}\right)+\Theta\left(\rho-\rho_{\beta}\right)\right],
\end{aligned}
$$

where $\lambda_{\alpha \beta}=\lambda_{\alpha}-\lambda_{\beta}$.

Remembering the ordering of $\rho_{\alpha}$ we can now write the superpotential as

$$
\begin{gathered}
W=-2 \pi i R \sum_{\alpha<\beta}\left(c_{\alpha} s_{\beta}-c_{\beta} s_{\alpha}\right) \int d g_{\alpha} \wedge d g_{\beta}\left[\frac{\left(a_{\alpha}-a_{\beta}\right)}{\lambda_{\alpha \beta}^{2}} \Theta_{\alpha \beta}(\rho)\right. \\
\left.+\sum_{\gamma \neq \alpha, \beta}^{n} a_{\gamma}\left(\Lambda_{\alpha} \Theta\left(\rho-\rho_{\alpha}\right)+\Lambda_{\beta} \Theta\left(\rho-\rho_{\beta}\right)+\Lambda_{\gamma} \Theta\left(\rho-\rho_{\gamma}\right)\right)\right],
\end{gathered}
$$

where $\Theta_{\alpha \beta}(\rho)$ is the step function that is 1 between $\rho_{\alpha}$ and $\rho_{\beta}$ and 0 otherwise. We can also evaluate the rest of the integral. From the construction of the homotopy functions we notice following fact,

$$
\int_{C_{\gamma}} g_{\alpha} d g_{\beta}=\delta_{\alpha \gamma}
$$

where $C_{\gamma}$ is a line along the $\rho=\rho_{\gamma}$ as $\sigma$ varies from 0 to 1 . By this and the Stokes' theorem it is easy to evaluate the integral

$$
\int_{\gamma \delta} d g_{\alpha} \wedge d g_{\beta}=\frac{1}{2}\left(\delta_{\alpha \gamma}+\delta_{\beta \delta}-\delta_{\alpha \delta}-\delta_{\beta \gamma}\right)
$$

where the integral is over a band of the strip defined by $\rho_{\delta}<\rho<\rho_{\gamma}, 0 \leq \sigma \leq 1$. Thus, the final result is

$$
W=-2 \pi i R \sum_{\alpha<\beta}\left(c_{\alpha} s_{\beta}-c_{\beta} s_{\alpha}\right)\left[\frac{\left(a_{\alpha}-a_{\beta}\right)}{\lambda_{\alpha \beta}^{2}}+\frac{1}{2} \frac{1}{\lambda_{\alpha \beta}} \sum_{\gamma \neq \alpha, \beta} a_{\gamma}\left(\frac{1}{\lambda_{\gamma \alpha}}+\frac{1}{\lambda_{\gamma \beta}}\right)\right],
$$

where $\alpha, \beta, \gamma$ run from 0 to $n$.

If we choose the parametrization where first NS fivebrane is identified with the large $\lambda$ region, it is given by

$$
v=\lambda+\sum_{\alpha=1}^{n} \frac{c_{\alpha}}{\lambda-\lambda_{\alpha}}
$$




$$
\begin{aligned}
& w=\sum_{\alpha=1}^{n} \frac{s_{\alpha}}{\lambda-\lambda_{\alpha}}, \\
& t=\prod_{\alpha=1}^{n}\left(\lambda-\lambda_{\alpha}\right)^{-a_{\alpha}} .
\end{aligned}
$$

Notice that the $\alpha=0^{\text {th }}$ components in $w$ and $t$ are truncated. Sometimes, this parametrization makes the discussion of the physics more intuitive. For example, when we discuss the rotations of the branes, one of the branes must be fixed and this distinguished one is located at $\lambda=\infty$. Almost the same calculation gives us the superpotential,

$$
\begin{aligned}
W= & -2 \pi i R \sum_{\alpha<\beta}^{n}\left(c_{\alpha} s_{\beta}-c_{\beta} s_{\alpha}\right)\left[\frac{\left(a_{\alpha}-a_{\beta}\right)}{\lambda_{\alpha \beta}^{2}}+\frac{1}{2} \frac{1}{\lambda_{\alpha \beta}} \sum_{\gamma \neq \alpha, \beta} a_{\gamma}\left(\frac{1}{\lambda_{\gamma \alpha}}+\frac{1}{\lambda_{\gamma \beta}}\right)\right] \\
& -2 \pi i R\left[\left(\sum_{\gamma=1}^{n} a_{\gamma}\right)\left(\sum_{\beta=1}^{n} s_{\beta}\right)-\frac{1}{2} \sum_{\gamma \neq \beta} \frac{a_{\gamma} \lambda_{\gamma} s_{\beta}}{\lambda_{\gamma \beta}}\right] .
\end{aligned}
$$

Here $\alpha, \beta, \gamma$ run from 1 to $n$.

The above discussion can easily be generalized to the cases with $N_{f}$ hypermultiplets, corresponding to $N_{f} \mathrm{D}$ sixbranes or semi-infinite $\mathrm{D}$ fourbranes. To illustrate this let us consider a situation where the $N_{f}$ of semi-infinite D fourbranes are attached to the right hand side of the second NS fivebrane. The curve for such a configuration had already been written down 14. In the parametric form, it is given by

$$
\begin{aligned}
& v=\frac{\left(\lambda-\lambda_{+}\right)\left(\lambda-\lambda_{-}\right)}{\mu \lambda}, \\
& w=\lambda, \\
& t=\mu^{-N_{c}} \lambda^{N_{c}-N_{f}}\left(\lambda-\lambda_{+}\right)^{r}\left(\lambda-\lambda_{-}\right)^{N_{f}-r},
\end{aligned}
$$

where $\lambda_{ \pm}$are the two solutions of $v=0$, and $\mu$ is bare mass for the adjoint chiral multiplet. Employing the same method as above, we get following superpotential:

$$
\begin{aligned}
W & =2 \pi i \frac{R \lambda_{+} \lambda_{-}}{\mu}\left[2\left(N_{c}-N_{f}\right)+\left(N_{f}-r\right)+r\right], \\
& =2 \pi i \frac{R \lambda_{+} \lambda_{-}}{\mu}\left(2 N_{c}-N_{f}\right) .
\end{aligned}
$$

This precisely agrees with the minimum value of $N=1$ gauge theory superpotential 
$\left(N_{f}<N_{c}\right)$ given as follows[20, 14];

$$
W_{\text {eff }}=\left(N_{c}-N_{f}\right)\left(\frac{\Lambda_{N=1}^{3 N_{c}-N_{f}}}{\operatorname{det} M}\right)^{1 /\left(N_{c}-N_{f}\right)}+\frac{1}{2 \mu}\left(\operatorname{Tr}\left(M^{2}\right)-\frac{1}{N_{c}}(\operatorname{Tr} M)^{2}\right),
$$

where $\Lambda_{N=1}$ is the dynamical scale of $N=1$ SQCD. More explicitly,

$$
W_{\text {Gauge-theory }}=-\frac{1}{4 \pi i} W_{\mathrm{M}-\text { theory }}
$$

with the identification $\lambda_{ \pm}=m_{ \pm}$, where $m_{ \pm}$are the only two possibly different eigenvalues of the meson matrix $M$ whose elements are $\tilde{Q}_{i}^{a} Q_{b}^{i}$. This is the first concrete evidence that Witten's proposal for the superpotential is correct. There is an explicit rotation angle dependence through $\mu \sim \tan \theta$. The dependence on $r$ comes only through $\lambda_{ \pm}$.

\section{Discussion}

In this paper we found a parametric representation of the brane configurations corresponding to the $N=1$ SQCD. Extending the Witten's method, we developed a general formalism to calculate the superpotentials and compared with known gauge theory results.

The comparison with gauge theory result might be interesting when we consider gauge theories for product groups. As mentioned earlier, one motivation for this comes from the study of configurations of $k$ 'coincident' NS fivebranes linked to an 'coincident' NS fivebrane linked by $N_{c}$ D fourbranes, and have the form of the superpotential for that configuration[0]. The gauge theory has $S U\left(N_{c}\right)$ gauge symmetry. Now when the NS fivebranes separate it corresponds to, in the field theory, resolution

of the singularity in the superpotential of the form $W=X^{k+1}+$ lower powers 22. Then the gauge symmetry is spontaneously broken to $S U\left(N_{c}\right) \rightarrow \prod S U\left(r_{i}\right)$, where $\sum_{i} r_{i}=N_{c}$. Of course the detailed form of the potential depends on the deformation parameters away from the coincident limit.

Also more thorough understanding is necessary on the role of the complex volume form which appears in the definition of the superpotential. The implication 
of the superpotential in relation to the symmetry enhancement when the branes collapse is not fully understood. These are currently being investigated.

\section{Acknowledgement}

KhO would like to thank POSTECH, SJS and SN to APCTP for hospitality. SJS also likes to thank APCTP for financial support and PIMS for the hospitality during his stay at UBC where part of the work is done. This work is supported in part by Ministry of Education(BSRI-96-2441 for SJS, BSRI-97-2442 for SN), by KOSEF (971-0201-001-2 for SJS, 961-0201-001-2 for SN), and by CTP/SNU through the SRC program of $\operatorname{KOSEF}(\mathrm{SN})$. Finally, we would like to thank S. Hyun for his participation in the early stage.

\section{References}

[1] J. Polchinski, S. Chaudhuri and C.V. Johnson, "Notes On D -Branes," hepth/9602052, J. Polchinski, "TASI Lectures On D-Branes," hep-th/9611050.

[2] A. Hanany and E. Witten, "Type IIB Superstrings, BPS Monopoles, and ThreeDimensional Gauge Dynamics", Nucl. Phys. B492 (1997) 152, hep-th/9611230.

[3] J. de Boer, K. Hori, Y. Oz and Z. Yin, "Branes and Mirror Symmetry in $N=2$ Supersymmetric Gauge Theories in Three Dimensions," hep-th/9702154.

[4] S. Elitzur, A. Giveon and D. Kutasov, "Branes and $N=1$ Duality in String Theory", Phys. Lett. B400 (1997) 269, hep-th/9702014.

[5] S. Elitzur, A. Giveon, D. Kutasov, E. Rabinovici and A. Schwimmer, "Brane Dynamics and $N=1$ Supersymmetric Gauge Theory", hep-th/9704104.

[6] N. Evans, C.V. Johnson and A.D. Shapere, "Orientifolds, Branes, and Duality of 4D Gauge Theories", hep-th/9703210. 
[7] J. H. Brodie and A. Hanany, "Type IIA Superstrings, Chiral Symmetry, and $N=14$ D Gauge Theory Dualities", hep-th/9704043.

[8] R. Tartar, "Dualities in 4D Theories with Product Gauge Groups from Brane Configurations", hep-th/9704198.

[9] A. Marshakov, M. Martellini, and A. Morozov, "Insights and Puzzles from Branes: 4D SUSY Yang-Mills from 6D Models," hep-th/9706060.

[10] K. Landsteiner, E. Lopez, and D.A. Lowe, " $N=2$ Supersymmetric Gauge Theories, Branes, and Orientifolds," hep-th/9705199.

[11] A. Gorsky, S. Gukov, and A. Mironov, "Multiscale $N=2$ SUSY field theories, integrable systems and their stringy/brane origin - I", hep-th/9707120.

[12] E. Witten, "Solutions of Four-Dimensional Field Theories via M Theory", hepth/9703166.

[13] A. Brandhuber, J. Sonnenschein, S. Theisen and S. Yankielowicz, "M Theory and Seiberg-Witten Curves: Orthogonal and Symplectic groups", hep-th/9705232.

[14] K. Hori, H. Ooguri and Y. Oz, "Strong Coupling Dynamics of Four-Dimensional $N=1$ Gauge Theories from M Theory Fivebrane", hep-th/9706082.

[15] E. Witten, "Branes and the Dynamics of QCD," hep-th/9706109.

[16] A. Brandhuber, N. Itzhaki, V. Kaplunovsky, J. Sonnenschein and S. Yankielowicz, "Comments on the M-theory Approach to $N=1$ SQCD and Brane Dynamics", hep-th/9706127.

[17] N. Seiberg and E. Witten, "Electric-Magnetic Duality, Monopole Condensation, and Confinement in $N=2$ Supersymmetric Yang-Mills Theory", Nucl. Phys. B426 (1994) 19, hep-th/9407087.

[18] N. Seiberg and E. Witten, "Monopoles, Duality and Chiral Symmetry Breaking in $N=2$ Supersymmetric QCD", Nucl. Phys. B431 (1994) 484, hep-th/9408099. 
[19] G. Dvali, M. Shifman, "Domain Walls in Strongly Coupled Theories," Phys. Lett. 396B (1997) 64, hep-th/9612128; A. Kovner, M. Shifman, A. Smilga, "Domain Walls in Supersymmetric Yang Mills Theories"; B. Chibisov and M. Shifman, "BPS-Saturated Walls in Supersymmetric Theories", hep-th/9706141.

[20] I. Affleck, M.Dine and N. Seiberg, "Dynamical Supersymmetry Breaking in Supersymmetric QCD," Nucl. Phys. B241 (1984) 493.

[21] J. L. F. Barbón, "Rotated Branes and $N=1$ Duality", Phys. Lett. 402B (1997) 59, hep-th/9703051.

[22] D. Kutasov, "Comments on Duality in $N=1$ Supersymmetric Nonabelian Gauge Theories", Phys. Lett. 351B (1995) 230, hep-th/9503086; D. Kutasov and A. Schwimmer, "On Duality in Supersymmetric Yang Mills Theory", Phys. Lett. 354B (1995) 315, hep-th/9505004; D. Kutasov, A. Schwimmer, and N. Seiberg, "Chiral Rings, Singularity Theory, and Electric-Magnetic Duality", Nucl. Phys. B459 (1996) 455, hep-th/9510222. 